\title{
Surgical treatment of nail bed subungual exostosis
}

\author{
Melih Malkoc ${ }^{1}$, MD, Ozgur Korkmaz ${ }^{1}$, MD, Mert Keskinbora' ${ }^{1}$ MD, Ali Seker ${ }^{1}$, MD, Ismail Oltulu ${ }^{1}$, MD, \\ Ahmet Murat $\underline{\text { Bulbul }}^{1}$, MD, Ferhat $\underline{\text { Say }}^{2}$, MD, Aslı $\underline{\text { Cakir }}^{3}$, MD
}

INTRODUCTION A subungual exostosis (SE) is a bony overgrowth that is permanently attached to the tip of the distal phalanx. Its pathology differs from osteocartilaginous exostoses in that it mainly involves the overgrowth of normal bone, which may present beneath the toenail or on the sides of the toe. This retrospective study aimed to report the results of surgical treatment when the diagnosis of SE was delayed; the condition was initially considered to be another pathology affecting a different nail or the terminal toe.

METHODS A total of 17 patients ( 12 female, five male) were included in the study. All surgical resections were performed by the same surgeon using the same surgical technique, with the patient under digital anaesthesia. The patients were evaluated pre- and postoperatively (on Weeks 1 and 6, the first year, and the last follow-up visit) using the American Orthopaedic Foot and Ankle Society questionnaire and the Visual Analogue Scale score.

RESULTS The patients underwent surgery for SE removal between December 2009 and October 2012. Their mean age was $21.3 \pm 4.4$ (range 14-29) years and the mean follow-up period was $27.1 \pm 7.8$ (range 18-45) months. Clinical or radiological recurrence was not observed in any of the patients during the follow-up period. Four patients had superficial infections, which were treated using appropriate antibiotic therapies.

CONCLUSION As SE is an uncommon benign lesion, its diagnosis may be delayed. Radiography may be useful in obtaining a differential diagnosis.

Keywords: nail bed, nail tip pathology, subungual exostosis, tumour

\section{INTRODUCTION}

A subungual exostosis (SE) is a bony overgrowth that is permanently attached to the tip of the distal phalanx. It was first described by Dupuytren, who observed SE on toes; ${ }^{(1)}$ Hutchinson later reported SE on fingertips. ${ }^{(2)}$ The pathology of SE differs from that of osteocartilaginous exostoses. SE usually involves the overgrowth of normal bone, which may occur beneath the toenail or on the side of the toe, and can obstruct nail growth. ${ }^{(3)}$ While chronic irritation is thought to be the cause of metaplasia in fibrocartilage, the pathogenesis remains unknown. According to the literature, trauma, chronic infection, tumour, hereditary anomalies and the activation of a cartilaginous cyst are all possible causes. ${ }^{(4)}$ Some studies have reported that trauma is the main contributing factor in the development of SE, with subsequent acute and chronic inflammation causing cartilaginous metaplasia. ${ }^{(5,6)}$ An alternative view is that chronic infection is the result and not the cause of SE. ${ }^{(6)}$

SE is most commonly diagnosed in children and young adults; most of these lesions are located in the big toe, although they can occur (albeit infrequently) in other toes. ${ }^{(3)}$ Only a small percentage of these lesions occur in the fingers. ${ }^{(4)}$ Regarding its pathology, SE commonly presents as swelling beneath the nail, causing separation between the nail and toe. It is frequently misdiagnosed and incorrectly treated.

Microscopic findings include trabecular bone formation at the basement layer and a proliferating fibrocartilaginous cap. Immature SE usually involves a thick cartilaginous layer, while mature lesions have a thin cartilaginous layer. Mature lesions consist mainly of trabecular bone tissue. The cartilaginous cap includes mitotic hypercellularity and dense nuclei. Although these findings suggest malignancy, the lack of anaplasia indicates that the lesions are benign. ${ }^{(7)}$ The differential diagnosis includes a wide range of benign and malignant tumours, skin lesions, chronic infections, soft tissue and nail pathologies. Radiological findings generally provide sufficient information for diagnosis. ${ }^{(3)}$ Magnetic resonance imaging (MRI) is helpful in the diagnosis of SE due to its ability to detect the different signal formations of tumours. ${ }^{(8-10)}$

The present study aimed to report the results of surgical treatment administered in cases where the diagnosis of SE was delayed. In these cases, SE was initially considered to be another pathology affecting a different nail or the terminal toe (e.g. a wart, subungual infection, and nail or skin pathologies), and did not improve following various treatment methods (e.g. systemic and topical antifungal drugs, antibiotics, topical salicylic acid, and cauterisation). We also aimed to highlight the importance of careful excision of SE to prevent local recurrence, and careful repair of the nail bed to ensure better outcomes.

\section{METHODS}

This retrospective study was approved by the ethics board of our institution and conducted in accordance with the Declaration of Helsinki. Informed written consent was obtained from all patients included in the study. Patients who underwent surgery for SE on the toe (Fig. 1) between December 2009 and October 2012 at

${ }^{1}$ Department of Orthopedics and Traumatology, School of Medicine, Istanbul Medipol University, ${ }^{2}$ Department of Orthopedics and Traumatology, Faculty of Medicine, Ondokuz Mayıs University, ${ }^{3}$ Department of Pathology, School of Medicine, Istanbul Medipol University, Turkey

Correspondence: Dr Melih Malkoc, Specialist-Orthopedic Surgeon, Department of Orthopedics and Traumatology, Istanbul Medipol University, School of Medicine, Tem Avrupa Otoyolu Goztepe Cıkısi, No. 1 Bagcilar 34214, Istanbul, Turkey.memalkoc@yahoo.com 


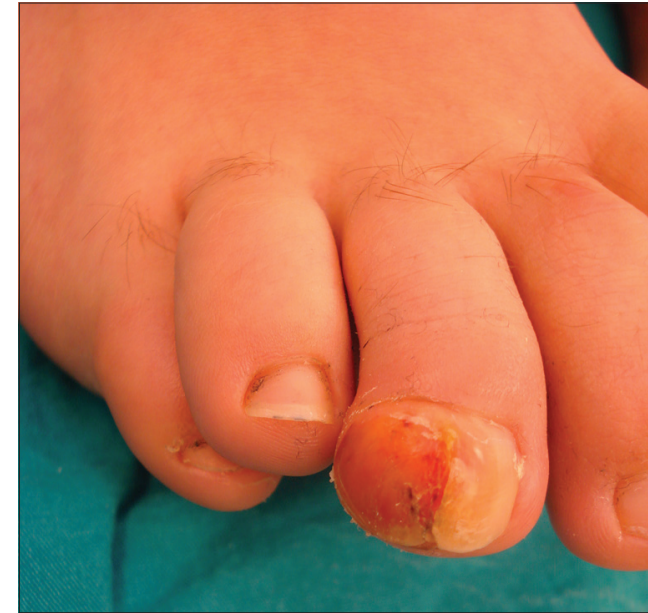

Fig. 1 Photograph shows the clinical presentation of a subungual exostosis located in the third toe of a right foot.
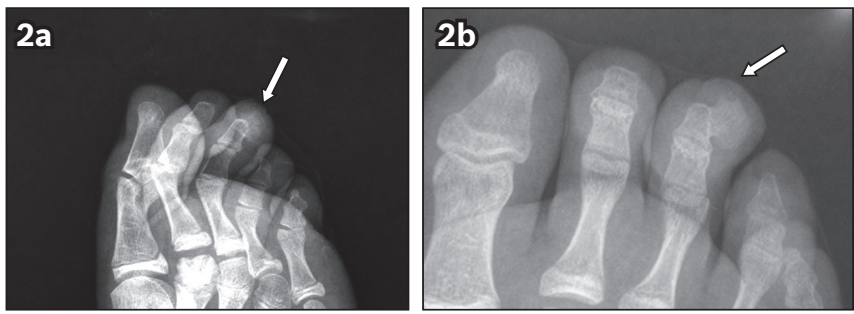

Fig. 2 (a) Lateral and (b) anteroposterior radiographs of the right foot show a subungual exostosis (arrows) in the third toe.

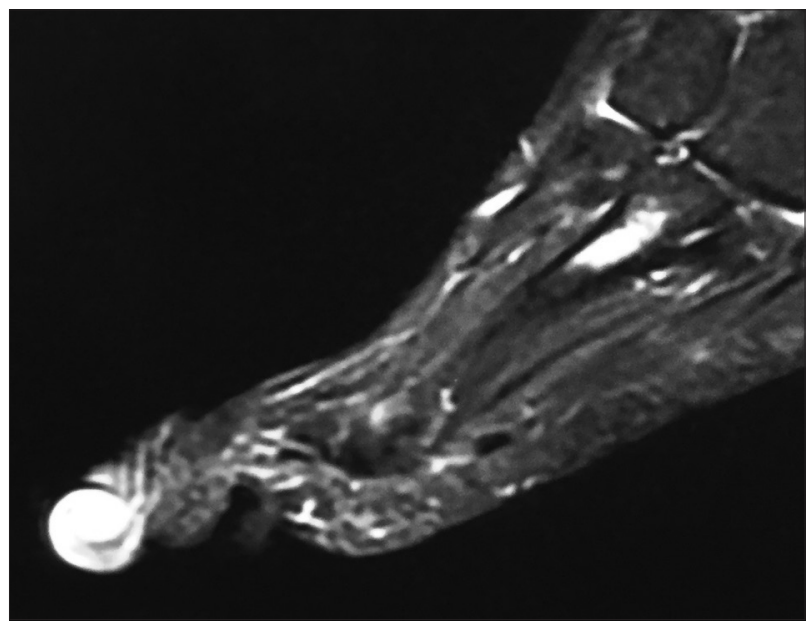

Fig. 3 Sagittal T1 MR image of the right foot shows a subungual exostosis in the third toe.

Istanbul Medipol University, Istanbul, Turkey, were eligible for inclusion in the study.

After excluding patients who had concomitant benign or malignant foot tumours and patients who did not attend any follow-up sessions after surgery, a total of 17 patients ( 12 female, five male) were included in the study. All patients were evaluated pre- and postoperatively (on Weeks 1 and 6, the first year, and the last follow-up visit) using the American Orthopaedic Foot and Ankle Society (AOFAS) questionnaire and the Visual Analogue Scale (VAS) score. Standard anteroposterior and lateral weight-bearing radiographs, and MR images were obtained preoperatively (Figs. $2 \& 3$ ). After surgery, the patients' progress
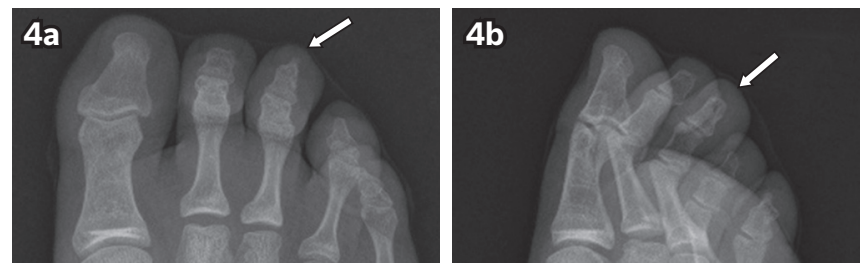

Fig. 4 Postoperative (a) lateral and (b) anteroposterior radiographs of the right foot show successful excision of the subungual exostosis (arrows) in the third toe.

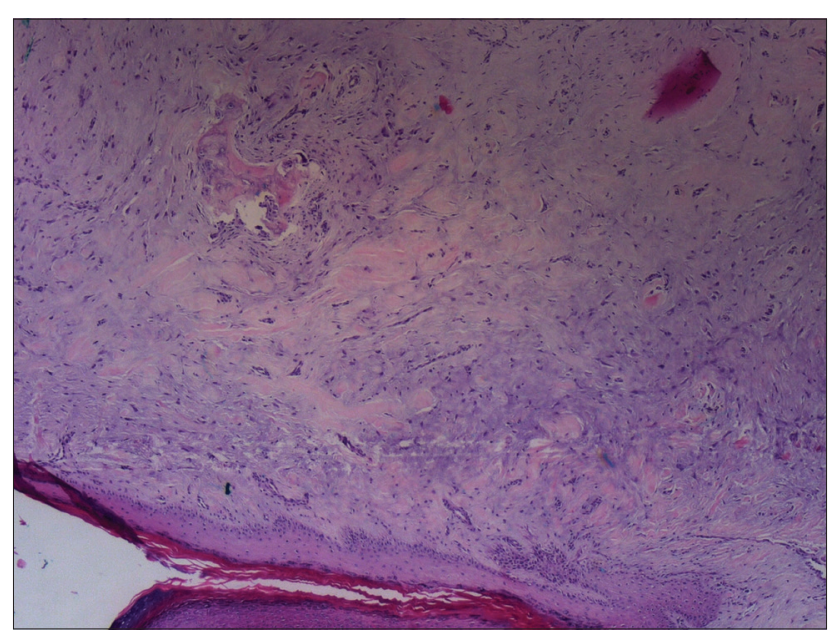

Fig. 5 Photomicrograph shows new bone and bone-forming cells (i.e. osteoblasts) in the chondromyxoid stroma under the keratinised epithelium (Haematoxylin \& eosin, × 200).

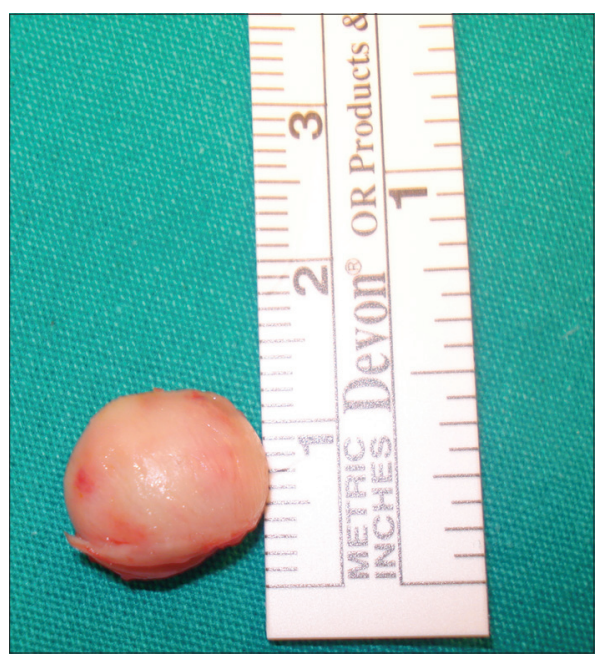

Fig. 6 Photograph shows the excised mass.

was monitored with radiography during the follow-up visits (Fig. 4). They were evaluated with MRI during the follow-up visit one year after surgery.

All surgical resections were performed by the same surgeon using the same surgical technique. During the surgical resections, the patients were placed under digital anaesthesia with a digital tourniquet. In each case, the toenail was partially removed, as the mass had protruded from the nail bed. The lesion was removed using an L-shaped incision that was longitudinal on the nail bed and transverse on the tip. The mass was excised together with the pedicle from the dorsal surface of the distal phalanx and the excised lesions were sent for pathological evaluation (Figs. 5 \& 6). 
Table I. Demographics of the patients with subungual exostosis (SE).

\begin{tabular}{|c|c|c|c|c|c|c|}
\hline No. & Age (yr) & Gender & Affected toe & Previous diagnosis & $\begin{array}{l}\text { Time from presentation } \\
\text { to diagnosis of SE (mth) }\end{array}$ & Follow-up period (mth) \\
\hline 1 & 14 & Female & 1 & Onychomycosis & 8 & 34 \\
\hline 3 & 21 & Male & 3 & Onychomycosis & 7 & 45 \\
\hline 4 & 17 & Female & 1 & Verruca & 10 & 32 \\
\hline 5 & 25 & Female & 1 & Onychomycosis & 12 & 21 \\
\hline 6 & 16 & Female & 1 & Onychomycosis & 6 & 19 \\
\hline 7 & 24 & Female & 3 & Granuloma pyogenicum & 7 & 27 \\
\hline 8 & 21 & Male & 1 & Verruca & 3 & 32 \\
\hline 9 & 17 & Male & 1 & Onychomycosis & 12 & 23 \\
\hline 10 & 26 & Female & 1 & Onychomycosis & 8 & 25 \\
\hline 11 & 19 & Female & 1 & Granuloma pyogenicum & 3 & 32 \\
\hline 13 & 22 & Female & 1 & Granuloma pyogenicum & 3 & 19 \\
\hline 14 & 27 & Female & 3 & Onychomycosis & 7 & 23 \\
\hline 15 & 16 & Male & 1 & Verruca & 6 & 21 \\
\hline 16 & 29 & Female & 2 & Verruca & 5 & 19 \\
\hline 17 & 23 & Female & 1 & Onychomycosis & 9 & 36 \\
\hline
\end{tabular}

After the removal of the lesion, the damaged nail bed was carefully repaired using a 6-0 absorbable suture and the excised nail was fixed onto its bed. Intraoperative fluoroscopy was not used in all the operations.

\section{RESULTS}

The mean age of the patients was $21.3 \pm 4.4$ (range 14-29) years and the mean follow-up period was $27.1 \pm 7.8$ (range 18-45) months. The mean duration of the disease before surgery was $6.8 \pm 2.7$ (range 3-12) months. Before the surgery for SE, nine patients had been treated for onychomycosis, four for granuloma pyogenicum and four for verruca. Radiography was not performed for the patients before they presented at our institution. Table I shows the patient demographics.

Neither clinical nor radiological recurrence was observed in any of the 17 patients during the follow-up period. All patients returned to their daily activities without any disability after surgical treatment. Four patients had superficial infections, which were treated using appropriate antibiotic therapies. The mean VAS score was $8.1 \pm 1.1$ (range $6-10$ ) before surgery and $0.7 \pm 0.5$ (range 0-2) after surgery at the last follow-up visit. The mean AOFAS score was $36.0 \pm 4.9$ (range 31-46) before surgery and $93.6 \pm 2.3$ (range 89-97) after surgery. Pathological evaluation confirmed the diagnosis of SE in all 17 cases.

\section{DISCUSSION}

Due to the close-fitting and narrow structure $(1-2 \mathrm{~mm}$ ) of the subungual space, any type of occupying lesion may disturb the distal phalanx and cause pain. ${ }^{(11)}$ Reports on the misdiagnosis and incorrect treatment, or delayed diagnosis and treatment, of SE are common in the literature. ${ }^{(12-16)}$ In the present study, all 17 patients with SE received different clinical diagnoses and underwent other primary treatments before receiving treatment for SE. The use of radiography may be helpful in the differential diagnosis of SE.
SE is most frequently seen in the big toe. However, it can also present in the other toes and fingers, although the incidence of the latter is low. ${ }^{(12,17)}$ It is generally located on the dorsomedial aspect of the nail bed. ${ }^{(18)}$ In the present study, SE was found in the big toe of 12 patients and in the other toes of five patients. This finding is consistent with the current literature, although only four of the 17 patients in the present study had lesions on the dorsolateral aspect of the toe. SE is commonly diagnosed during adolescence and has a female preponderance. ${ }^{(19)}$ This was observed in the present study, where 12 of the 17 patients were female. The age distribution of the patients in the present study was also consistent with that described in the literature. ${ }^{(18)}$

SE generally presents with swelling of the digit, ulceration, infection of the nail bed and secondary changes in the surrounding tissues. ${ }^{(7)}$ It appears as a solid, white, subungually-located, pigmented, haemangiomatous mass in the early stages. ${ }^{(20)}$ As the mass grows, local tissue indurations and onycholysis may occur. Paronychia is observed in some patients. ${ }^{(21)}$ The nail, which was initially elevated, detaches, resulting in the formation of fibrous tissue on its surface; soft tissue has the potential to erode. ${ }^{(22)}$ We did not detect paronychia in any of our patients. However, all of them had a detached nail, with a fibrous tissue mass and pain upon weight-bearing.

The main objective of surgical treatment of SE is to excise the exostosis up to the normal bone tissue border, in order to prevent recurrence and avoid nail deformities by protecting the digit matrix. ${ }^{(17)}$ In this study, we repaired the nail bed of the exposed nail and protected the unexposed nail. No signs of recurrence were detected on follow-up (mean duration 27.1 months). In $\mathrm{SE}$, pain results from the presence of bulky lesions in the tight subungual space ${ }^{(11)}$ and from pressure on the nail bed due to the growing exostosis when walking. ${ }^{(22)}$ All of the patients in the present study only complained of pain during the preoperative period. 
In conclusion, SE is a benign and uncommon lesion that is infrequently noticed by physicians. This may result in delays in diagnosis and treatment. Therefore, it is important that SE is included in the differential diagnosis of toe-tip pathologies. Early suspicion of SE should lead to radiography to verify the diagnosis and prompt surgical excision if the SE is confirmed. Radiography may be useful in the differential diagnosis of SE, while local excision of the mass with the overlying fibrocartilaginous cap is the preferred treatment. Removal of adjacent unaffected bone may prevent local recurrence, and repair of the nail bed can provide better outcomes.

\section{REFERENCES}

1. Dupuytren G, Le Gros Clark F, eds. On the Injuries and Diseases of Bones: Being Selections from the Collected Edition of the Clinical Lectures of Baron Dupuytren. London: Sydenham Society, 1847: 408-10.

2. Hutchinson J. Subungual exostosis of the great toe: excision with success. Lancet $1857 ; 2: 246-7$.

3. Evison G, Price CH. Subungual exostosis. Br J Radiol 1966; 39:451-5.

4. Carroll RE, Chance JT, Inan Y. Subungual exostosis in the hand. J Hand Surg Br 1992; 17:569-74.

5. Ilyas W, Geskin L, Joseph AK, Seraly MP. Subungual exostosis of the third toe. J Am Acad Dermatol 2001; 45(6 Suppl):S200-1.

6. Landon GC, Johnson KA, Dahlin DC. Subungual exostoses. J Bone Joint Surg Am 1979; 61:256-9.

7. Resnick D, Kyriakos M, Greenway GD. Tumors and tumor-like lesions of bone: imaging and pathology of specific lesions. In: Resnick D, eds. Diagnosis of Bone and Joint Disorders. Philadelphia: WB Saunders Company, 1995: 3628-938.

8. Horcajadas AB, Lafuente JL, de la Cruz Burgos R, et al. Ultrasound and MR findings in tumor and tumor-like lesions of the fingers. Eur Radiol 2003; 13:672-85.

9. Theumann NH, Goettmann S, Le Viet D, et al. Recurrent glomus tumors of fingertips: MR imaging evaluation. Radiology 2002; 223:143-51.

10. Teh J, Whiteley G. MRI of soft tissue masses of the hand and wrist. Br J Radiol 2007; 80:47-63.

11. Fornage BD. Glomus tumors in the fingers: diagnosis with US. Radiology 1988; 167:183-5.

12. Tuzuner T, Kavak A, Parlak AH, Ustundag N. Subungual osteochondroma: a diagnostic dilemma. J Am Podiatr Med Assoc 2006; 96:154-7.

13. Campanelli A, Borradori L. Images in clinical medicine. Subungual exostosis. N Engl J Med 2008; 359:e31.

14. Stănescu L, Popescu CF, Niculescu CE, et al. Subungual exostosis of the big toe. Rom J Morphol Embryol 2009; 50:501-3.

15. Singh R, Jain M, Goel R, et al. Subungual exostosis of the great toe: a case report and tumor overview. Foot Ankle Spec 2011; 4:376-8.

16. García Carmona FJ, Pascual Huerta J, Fernández Morato D. A proposed subungual exostosis clinical classification and treatment plan. J Am Podiatr Med Assoc 2009; 99:519-24.

17. de Berker DA, Baran R. Disorders of nails. Subungual exostosis. In: Burns T, Breathnach S, Cox N, Griffiths C, eds. Rook's Textbook of Dermatology. Chichester: A John Wiley and Sons Ltd Publication, 2010: 65.33-65.34.

18. Baek HJ, Lee SJ, Cho KH, et al. Subungual tumors: clinicopathologic correlation with US and MR imaging findings. Radiographics 2010; 30:1621-36.

19. Vázquez-Flores H, Domínguez-Cherit J, Vega-Memije ME, Sáez-DeOcariz M. Subungual osteochondroma: clinical and radiologic features and treatment. Dermatol Surg 2004; 30:1031-4.

20. Jetmalani SN, Rich P, White CR Jr. Painful solitary subungual nodule. Subungual exostosis (SE). Arch Dermatol 1992; 128:849, 852.

21. Miller-Breslow A, Dorfman HD. Dupuytren's (subungual) exostosis. Am J Surg Pathol 1988; 12:368-78.

22. Aggarwal K, Gupta S, Jain VK, Mital A, Gupta S. Subungual exostosis. Indian J Dermatol Venereol Leprol 2008; 74:173-4 